\title{
Phosphofructokinase in rat lung during perinatal development: characterization of subunit composition and regulation by fructose 2,6-bisphosphate and glucose 1,6-bisphosphate
}

\author{
Ellen C. Heesbeen a , Gert Rijksen ${ }^{a}$, Joseph J. Batenburg ${ }^{b}$, \\ Lambert M.G. van Golde ${ }^{b}$ and Gerard E.J. Staal ${ }^{a}$ \\ a Department of Haematology, Laboratory of Medical Enzymology, University Hospital, \\ and ${ }^{b}$ Laboratory of Veterinary Biochemistry, Utrecht University, Utrecht (The Netherlands) \\ (Received 4 August 1986) \\ (Revised manuscript received 13 February 1987)
}

\begin{abstract}
Key words: Phosphofructokinase; Fructose 2,6-bisphosphate; Glucose 1,6-bisphosphate; Pulmonary surfactant; Development; (Rat lung)
\end{abstract}

The subunit composition of phosphofructokinase (ATP: D-fructose-6-phosphate-1-phosphotransferase, EC 2.7.1.11) was studied in rat lung during perinatal development. No change in subunit composition during this period was observed. The three subunits of phosphofructokinase $(L, M$ and $C$ ) were present in a ratio of approx. 65:25:10, respectively. In addition the levels of two effectors of phosphofructokinase were determined in rat lung during perinatal development: glucose 1,6-bisphosphate and fructose 2,6-bisphosphate. Until day 20 of gestation (term is 22 days) the glucose 1,6-bisphosphate level remains relatively constant (approx. $0.55 \mu \mathrm{mol} / \mathrm{g}$ protein), decreases before birth and increases sharply up to $1.04 \mu \mathrm{mol} / \mathrm{g}$ protein 2 days after birth. The amount of fructose 2,6-bisphosphate in rat lung shows a different developmental profile. A small peak is shown at day 17 of gestation whereas a larger peak up to 36.4 $\mathrm{nmol} / \mathrm{g}$ protein is shown at days 20 and 21 of gestation. The time of maximal fructose 2,6-bisphosphate content corresponds with the time of glycogen breakdown and acceleration of surfactant synthesis in prenatal rat lung. Both glucose 1,6-bisphosphate and fructose 2,6-bisphosphate stimulate lung phosphofructokinase. Half maximal stimulations occur in the range of 24.1-70.9 $\mu \mathrm{M}$ glucose 1,6-bisphosphate and 0.17-0.34 $\mu \mathrm{M}$ fructose 2,6-bisphosphate.

\section{Introduction}

Pulmonary surfactant is a lipid-protein complex that covers the alveolar surface. One of the

\footnotetext{
Abbreviation: Hepes, 4-(2-hydroxyethyl)-1-piperazineethanesulfonic acid.

Correspondence: E.C. Heesbeen, Department of Haematology, Division of Medical Enzymology, University Hospital, P.O. Box 16250, 3500 CG Utrecht, The Netherlands.
}

important features of pulmonary surfactant is that it provides a low surface tension at the air-liquid interface thus protecting the lung from alveolar collapse and transudation [1]. The synthesis of pulmonary surfactant (for review see Ref. 2) takes place in the alveolar type II epithelial cells and is accelerated considerably in the terminal part of gestation. Glycogen stored in the alveolar type II cells prior to the acceleration of surfactant synthesis is probably an important substrate for surfactant lipid synthesis [3-6]. Bourbon et al. [6] pro- 
vided direct evidence for the formation of saturated phosphatidylcholine from prelabeled endogenous glycogen in fetal rat lung. In a previous report we considered the possible role of glycolysis during the period of glycogen breakdown and onset of surfactant synthesis in rat lung [7]. The specific activity of one of the key enzymes of glycolysis phosphofructokinase, was found to be increased during this period. Phosphofructokinase catalyzes the phosphorylation of fructose 6-phosphate to fructose 1,6-bisphosphate; the enzyme is tetrameric with a molecular weight of about 330000 . Three types of phosphofructokinase subunits are known: $M$ (muscle), $L$ (liver) and $C$, recently reported to be present in rabbit [8] and rat [9] brain. Type $C$ is also known as type $F$ (fibroblast) [10] or P (platelet) [11]. Hybridization of the three subunits is possible. In this study we examined the subunit composition of phosphofructokinase during perinatal development in rat lung by means of SDS-polyacrylamide gel electrophoresis. In addition, the levels of glucose 1,6bisphosphate and fructose 2,6-bisphosphate were determined as was their influence on phosphofructokinase activity. Both bisphosphates are known effectors of phosphofructokinase. Glucose 1,6-bisphosphate acts not only as a stimulator of phosphofructokinase [12-14] but also as an inhibitor of hexokinase [13-15], which is another key enzyme of glycolysis. The dual role of this effector might be of importance in relation to glycogen breakdown and accelerated glycolysis during surfactant synthesis. The second effector examined, fructose 2,6-bisphosphate, is described as the most potent activator of phosphofructokinase $[16,17]$. Fructose 2,6-bisphosphate is found in many different tissues and is supposed to play an important role in control of glycolysis in liver $[16,18,19]$, hepatocytes $[20,21]$, fibroblasts [22], muscle [16] and pancreatic islets [23]. In this paper we report that both glucose 1,6-bisphosphate and fructose 2,6-bisphosphate can stimulate lung phosphofructokinase.

\section{Materials and Methods}

\section{Mațerials}

Glucose 1,6-bisphosphate, substrates and auxiliary enzymes for the determination of phos- phofructokinase activity were obtained from Boehringer (Mannheim, F.R.G.). Fructose 2,6-bisphosphate, dithiothreitol, $p$-aminobenzamidine, leupeptin and p-tosyl-1-lysine chloromethylketone- $\mathrm{HCl}$ were purchased from Sigma (St. Louis, MO, U.S.A.). SDS, acrylamide and bisacrylamide were purchased from Bio-Rad. Cibacron blue $\mathrm{F}_{3} \mathrm{GA}$ gel was obtained from Pierce Chemical $\mathrm{Co}$. (Rockford, IL, U.S.A.). The phosphofructokinase antiserum was a gift from Dr. Dunaway, Department of Pharmacology, Southern Illinois University School of Medicine, Springfield, IL, U.S.A.

\section{Animals and lung tissue}

Pregnant Wistar rats of known gestation times were purchased from the Central Institute for Breeding of Laboratory Animals in Zeist, The Netherlands. After killing the rat mothers $\left(\mathrm{CO}_{2}\right.$ inhalation) and decapitating the fetuses, fetal lungs were rapidly moved. Lungs of adult rats were perfused with saline to remove the red blood cells. Lung tissue used for the determination of fructose 2,6-bisphosphate and glucose 1,6-bisphosphate were stored in liquid nitrogen, those used for the characterization of phosphofructokinase subunits or kinetic studies were immediately homogenized in 3 vols. of ice-cold extraction buffer as described by Dunaway and Kasten [9] containing $50 \mathrm{mM}$ Tris-phosphate (pH 8.0)/25 mM NaF/1 mM ATP $/ 0.1 \mathrm{mM}$ EDTA/ $25 \mathrm{mM}\left(\mathrm{NH}_{4}\right)_{2} \mathrm{SO}_{4} / 10$ $\mathrm{mM}$ dithiothreitol/0.5 mM phenylmethylsulfonyl fluoride/1 $\mathrm{mM} p$-aminobenzamidine $/ 250 \mu \mathrm{g} / 1$ leupeptin/0.1 mM $p$-tosyl-1-lysine chloromethylketone- $\mathrm{HCl}$. The homogenate was centrifuged for $1 \mathrm{~h}$ at $100000 \times \mathrm{g}$ and the supernatant was stored at $-80^{\circ} \mathrm{C}$.

\section{Characterization of phosphofructokinase subunit compositions}

Lung supernatants prepared as described above were thawed quickly, heated for $3 \mathrm{~min}$ at $60^{\circ} \mathrm{C}$ and subsequently cooled in a $\mathrm{NaCl}$ ice bath to $4^{\circ} \mathrm{C}$. The solution was centrifuged for $30 \mathrm{~min}$ at $48000 \times g$ and $4^{\circ} \mathrm{C}$. The supernatant fluid was partially purified by Cibacron blue $\mathrm{F}_{3} \mathrm{GA}$ affinity chromatography as described by Dunaway and Kasten [9]. The eluted fraction containing phosphofructokinase activity was concentrated with an Amicon C30 microconcentrator and subjected to 
SDS electrophoresis in $6 \%$ polyacrylamide gels according to Laemmli [24]. The gels were silverstained as described by Wray et al. [25]. The quantification of the phosphofructokinase subunit bands was performed using a Gilford Response spectrophotometer with gel scanning accessory.

\section{Immunoblotting}

Proteins separated by SDS electrophoresis were transferred from un unfixed gel to a $0.45 \mu \mathrm{m}$ nitrocellulose sheet at $60 \mathrm{~V}$ for $2 \mathrm{~h}$ at $4^{\circ} \mathrm{C}$ using a Bio-Rad Trans-Blot apparatus. The transfer buffer contained $25 \mathrm{mM}$ Tris / $192 \mathrm{mM}$ glycine ( $\mathrm{pH} \mathrm{8.3)/}$ $20 \%(\mathrm{v} / \mathrm{v})$ methanol. The nitrocellulose sheet was blocked overnight in blocking solution containing $10 \mathrm{mM}$ Tris- $\mathrm{HCl}$ (pH 7.4)/0.9\% NaCl/0.05\% Tween 20. Subsequently the sheet was exposed to anti-phosphofructokinase serum in a $1: 1000$ dilution for $2 \mathrm{~h}$ at room temperature. The diluent was a $10 \mathrm{mM}$ Tris- $\mathrm{HCl}(\mathrm{pH} 7.4)$ solution with $0.9 \%$ $\mathrm{NaCl}$ and $1 \%$ gelatin. After washing the blot with blocking solution, horseradish peroxidase conjugated goat anti-rabbit IgG, diluted $1: 5000$ in the above diluent, was added and incubated for 90 min at room temperature. After washing with blocking solution the blot was visualized using a 4-chloro-1-naphthol staining solution [26].

Extraction and determination of glucose 1,6-bisphosphate and fructose 2,6-bisphosphate

$50-100 \mathrm{mg}$ of frozen lung tissue were powdered in a mortar cooled in liquid nitrogen. The powder was used for an alkaline extraction in 9 vols. of ice-cold $50 \mathrm{mM} \mathrm{NaOH}$. After neutralization with $1 \mathrm{M}$ acetic acid in $20 \mathrm{mM}$ Hepes, the extract was centrifuged for $10 \mathrm{~min}$ at $48000 \times g$ and $4^{\circ} \mathrm{C}$. The supernatant was collected quantitatively and kept on ice. To ensure the stability of glucose 1,6-bisphosphate and fructose 2,6-bisphosphate, the extracts were brought to a $\mathrm{pH}$ of 10 . Glucose 1,6 bisphosphate was assayed spectrophotometrically at $25^{\circ} \mathrm{C}$ according to Gerber et al. [27]. The glucose 1-phosphate used was essentially free of glucose 1,6-bisphosphate. Fructose 2,6-bisphosphate levels in the lung extracts were determined by stimulation of $\mathrm{PP}_{\mathrm{i}}$-phosphofructokinase of potato tubers according to Van Schaftingen et al. [18].
Assays of phosphofructokinase activity

The activity of the enzyme was assayed in a final volume of $1 \mathrm{ml}$ at $37^{\circ} \mathrm{C}$ in a Gilford Response spectrophotometer by measuring the decrease in absorbance at $340 \mathrm{~nm}$ in the enzyme coupled assay using 1.4 units aldolase, 1.5 units $s n$-glycerol-3-phosphate dehydrogenase, 4.5 units triosephosphate isomerase and $0.2 \mathrm{mM} \mathrm{NADH}$. Maximal velocities were measured in $100 \mathrm{mM}$ Tris- $\mathrm{HCl}$ buffer (pH 8.0) containing $10 \mathrm{mM} \mathrm{KCl}$, $5 \mathrm{mM} \mathrm{MgCl}_{2}, 5 \mathrm{mM}\left(\mathrm{NH}_{4}\right)_{2} \mathrm{SO}_{4}, 1 \mathrm{mM}$ EDTA, 5 $\mathrm{mM}$ dithiothreitol and $2 \mathrm{mM}$ fructose 6-phosphate. After incubation with sample, the reaction was started by the addition of $0.5 \mathrm{mM} \mathrm{MgATP}$. One unit of phosphofructokinase activity is defined as the amount of enzyme converting $1 \mu \mathrm{mol}$ fructose 6-phosphate into fructose 1,6-bisphosphate per min in the above system.

For regulatory studies the phosphofructokinase assays were performed at $37^{\circ} \mathrm{C}$ in $50 \mathrm{mM}$ Hepes (pH 7.1)/50 mM KCl/5 mM $\mathrm{MgCl}_{2} / 0.5 \mathrm{mM}$ $\left(\mathrm{NH}_{4}\right)_{2} \mathrm{SO}_{4} / 0.5 \mathrm{mM}$ EDTA $/ 1 \mathrm{mM}$ dithiothreitol, $0.04 \%$ bovine serum albumin $/ 0.2 \mathrm{mM}$ $\mathrm{NADH} / 1.4$ units aldolase/1.5 units $s n$-glycerol3-phosphate dehydrogenase/4.5 units triosephosphate isomerase $/ 1 \mathrm{mM}$ fructose 6-phosphate. The reaction was started by the addition of $1 \mathrm{mM}$ MgATP. The activity is expressed as $v / V_{\max }$ where $v$ is the activity in the latter assay conditions $(\mathrm{pH}$ 7.1) and $V_{\max }$ is the optimal activity determined in the assay described above at $\mathrm{pH}$ 8.0. Protein content was determined according to Bradford [28] using bovine serum albumin as a standard.

\section{Results}

\section{Phosphofructokinase subunit composition in rat lung} during perinatal development

Lung phosphofructokinase was partially purified using a heat step and Cibacron blue $F_{3} G A$ affinity chromatography. The overall recovery after Amicon concentration was approx. $60-80 \%$. The difference in recovery did not influence the phosphofructokinase subunit composition as proven by the following experiment. The same lung homogenate was partially purified with and without a heat step of $3 \mathrm{~min}$ at $60^{\circ} \mathrm{C}$. The overall recovery of the fraction with the heat step was approx. $25 \%$ lower than that of the partially purified fraction 


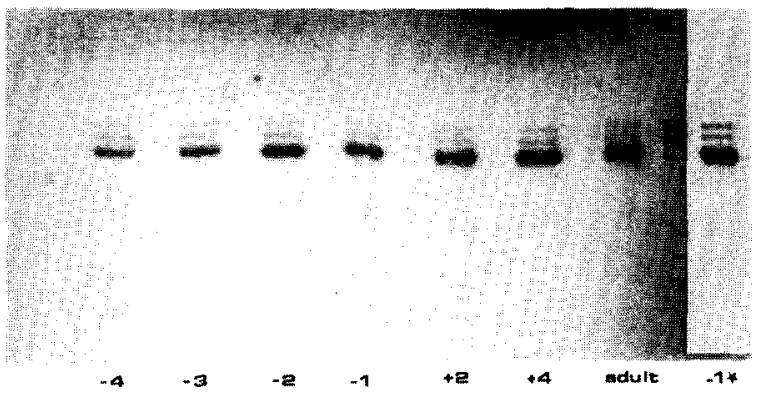

Fig. 1. SDS-polyacrylamide gel electrophoresis of partially purified lung phosphofructokinase. Phosphofructokinase samples of different days in rat lung development were subjected to SDS-polyacrylamide gel electrophoresis in a $6 \%$ polyacrylamide gel and were silver stained. Each lane contains $1 \mu \mathrm{g}$ of phosphofructokinase protein. The figure under each lane denotes the age in days relative to term. The far right lane $\left(-1^{*}\right)$ shows the results of immunoblotting studies with phosphofructokinase at day -1 , carried out as described in Materials and Methods.

without the heat step. The subunit composition of both fractions were identical. The heat step was necessary to get rid of disturbing proteins in the SDS electrophoresis and to enhance the reproducibility. The SDS electrophoresis of partially purified lung phosphofructokinase showed three phosphofructokinase subunits (Fig. 1): L, M and C with molecular weights of 80000,82500 and 86000 , respectively. These molecular weights are similar to those described by Dunaway and Kasten for a variety of other tissues $[9,29]$. The ratio of the three subunits (approx. 65\% L, 25\% $\mathrm{M}$ and $10 \%$ C) does not change during perinatal development (Fig. 2). The identity of phosphofructokinase was confirmed by immunoblotting with an antiphosphofructokinase serum (Fig. 1).

Levels of glucose 1,6-bisphosphate and fructose 2,6bisphosphate in rat lung during perinatal development

Glucose 1,6-bisphosphate and fructose 2,6-bisphosphate were determined in a $50 \mathrm{mM} \mathrm{NaOH}$ extract because both bisphosphates are acid labile and easily hydrolyse at low $\mathrm{pH}$ to glucose 6-phosphate and fructose 6-phosphate, respectively. The amount of glucose 1,6-bisphosphate remained relatively constant in the period before birth with a slight decrease just before term (Fig. 3). A 300\%

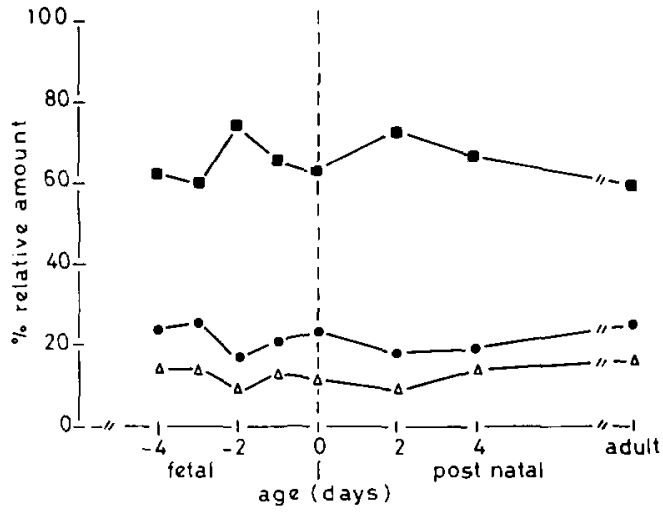

Fig. 2. Percentage of phosphofructokinase subunits $L$ (a), $M$ (•) and $C(\Delta)$ in rat lung during perinatal development. Each value in the curve represents the mean of three independent experiments.

increase is seen 2 days after birth with a maximum of $1.04 \pm 0.12 \mu \mathrm{mol}$ glucose 1,6-bisphosphate $/ \mathrm{g}$ protein. As the protein content of the rat lung at day 2 is approx. $44 \mathrm{mg}$ protein per gram wet weight [7], the average level of glucose 1,6-bisphosphate is $46 \mu \mathrm{mol} / \mathrm{kg}$ wet weight.

The fructose 2,6-bisphosphate curve shows no similarity to that of glucose 1,6-bisphosphate (Fig. 4); whereas glucose 1,6-bisphosphate peaks after birth, two peaks of fructose 2,6-bisphosphate appear before birth. Maximal levels of fructose 2,6-

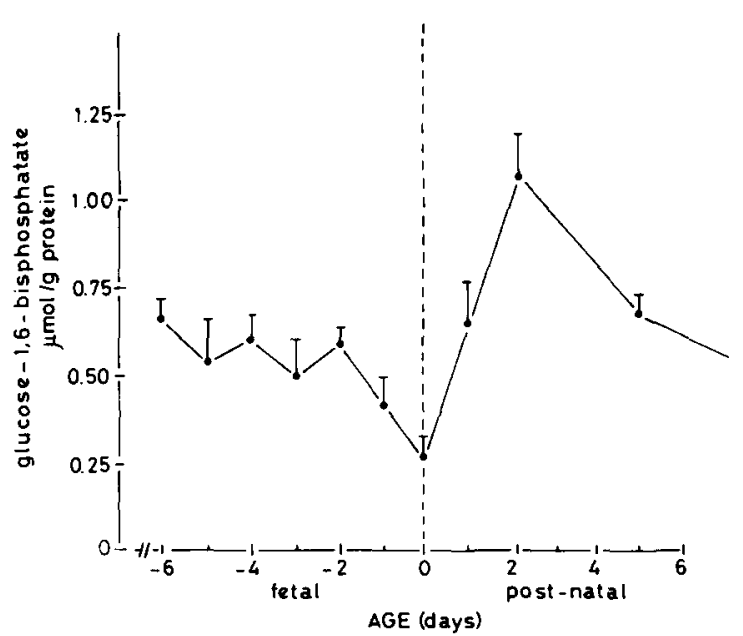

Fig. 3. Glucose 1,6-bisphosphate content of rat lung during perinatal development. Values are mean \pm S.E. of five experiments. 


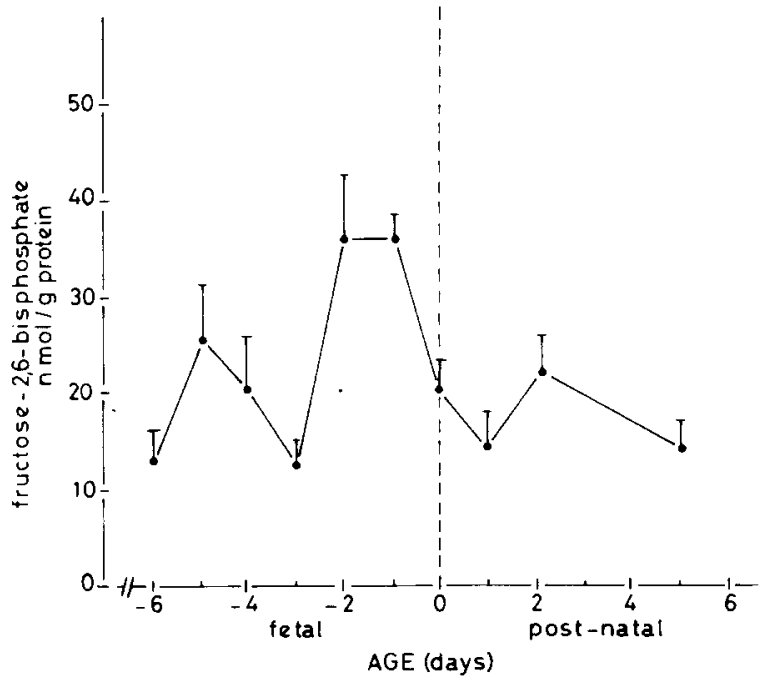

Fig. 4. Fructose 2,6-bisphosphate content of rat lung during perinatal development. Values are mean \pm S.E. of five experiments.

bisphosphate are reached at 2 and 1 days before birth $(36.0 \mathrm{nmol} / \mathrm{g}$ protein). After a minor increase 2 days after term, an adult value of approx. $23 \mathrm{nmol} / \mathrm{g}$ protein is reached.

Stimulation of rat lung phosphofructokinase by glucose 1,6-bisphosphate and fructose 2,6-bisphosphate

To remove endogenous glucose 1,6-bisphosphate, fructose 2,6-bisphosphate and other effectors, the lung extracts were thoroughly dialyzed against extraction buffer (see Materials and Methods), with omission of $1 \mathrm{mM}$ ATP. To remove any possibly contaminating fructose 2,6-bisphosphate in the fructose 6-phosphate solution which is used as a substrate in the phosphofructokinase activity assay, $\mathrm{HCl}$ was added to obtain a $\mathrm{pH}$ of 2 . The solution was heated for $30 \mathrm{~min}$ and subsequently neutralized with $\mathrm{NaOH}$. The excess of ammoniumsulfate in the auxiliary enzymes was removed by $\mathrm{G}-25$ gelfiltration.

The effect of fructose 2,6-bisphosphate and glucose 1,6-bisphosphate on lung phosphofructokinase was studied at three different times in perinatal development: 1 day before birth (day 1), 1 day after birth $($ day +1$)$ and 10 days after birth (day + 10). At $1.0 \mathrm{mM}$ ATP and $1.0 \mathrm{mM}$ fructose 6-phosphate ( $\mathrm{pH}$ 7.1), half maximal stimulations were obtained with $0.20 \mu \mathrm{M}$ fructose

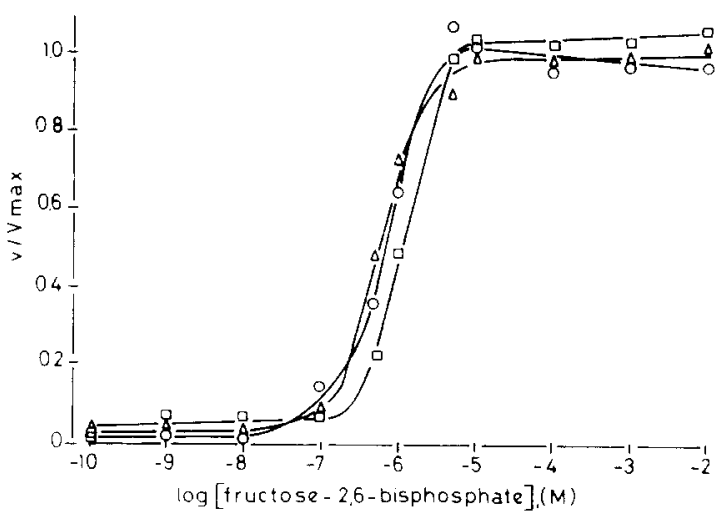

Fig. 5. Fructose 2,6-bisphosphate activation of rat lung phosphofructokinase at 1 day before birth $(O) ; 1$ day after birth $(\square)$ and 10 days after birth $(\Delta)$. Results are expressed as ratio of the maximal velocities $\left(v / V_{\max }\right)$ under the conditions described in Materials and Methods. Values are means of three independent experiments.

2,6-bisphosphate at day $-1,0.17 \mu \mathrm{M}$ fructose 2,6-bisphosphate at day +1 and $0.34 \mu \mathrm{M}$ fructose 2,6-bisphosphate at day +10 . Phosphofructokinase of all samples could be stimulated by fructose 2,6-bisphosphate to activities reached under optimal conditions, i.e., $V_{\max }$ (Fig. 5). Under identical conditions the effect of glucose 1,6-bisphosphate was investigated (Fig. 6). Half maximal stimulations were obtained at 24.1, 70.9 and 51.2 $\mu \mathrm{M}$ glucose 1,6-bisphosphate for day $-1,+1$ and

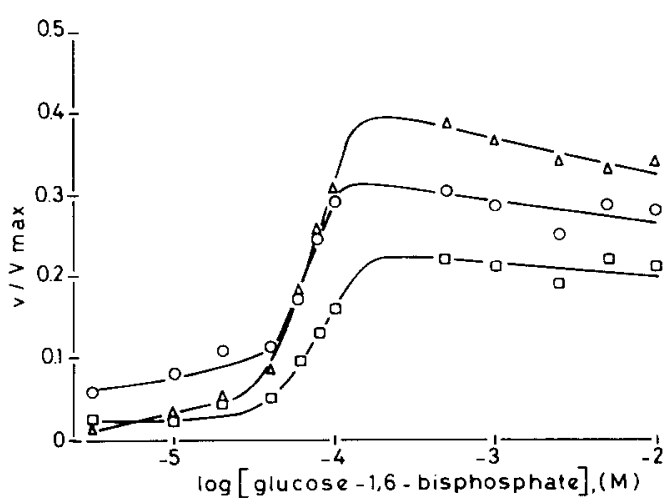

Fig. 6. Glucose 1,6-bisphosphate activation of rat lung phosphofructokinase at 1 day before birth $(O) ; 1$ day after birth $(\square)$ and 10 days after birth $(\triangle)$. Results are expressed as ratio of the maximal velocities $\left(v / V_{\max }\right)$ under the conditions described in Materials and Methods. Values are mean of two independent experiments. The variation between the two experiments was less than $10 \%$. 
+10 , respectively. Glucose 1,6-bisphosphate appears to be a weaker activator than fructose 2,6bisphosphate and stimulates phosphofructokinase only to $29 \%, 22 \%$ and $43 \%$ of $V_{\max }$ for day -1 , +1 and +10 , respectively.

\section{Discussion}

Up to now only little attention has been paid to the metabolic pathway between glucose 6-phosphate and dihydroxyacetonephosphate in studies on pulmonary surfactant synthesis during lung development. Glucose 6-phosphate is formed as an intermediate during glycogen breakdown and dihydroxyacetonephosphate is a glycolytic source for surfactant phospholipid synthesis. In a previous study [7] we showed that the specific activity of phosphofructokinase, the only key enzyme between glucose 6-phosphate and dihydroxyacetonephosphate, increased concomitantly with the onset of prenatal surfactant synthesis.

In this study we examined the role of two important effectors of phosphofructokinase activity, glucose 1,6-bisphosphate and fructose 2,6-bisphosphate. The effect of these bisphosphates on the phosphofructokinase activity depends on the isozyme composition of this enzyme [30,31]. Tsai and Kemp [32] showed by electrophoretic and immunoprecipitation data that rabbit lung phosphofructokinase is composed of $M$ and $L$ types and their hybrids. In contrast, our studies show that all three types of phosphofructokinase subunits are expressed in rat lung in a ratio $L / M / C$ of approx. $65: 25: 10$. The results also indicate that there is no change in subunit composition during lung development. Immunotitration studies with anti-sera against phosphofructokinase subunits $\mathrm{L}, \mathrm{M}$ and $\mathrm{C}$ also pointed at a constant $\mathrm{L}, \mathrm{M}$ and $C$ subunit ratio in rat lung during perinatal development (data not shown).

In view of these findings the following remarks can be made: (i) as hybridization is possible between the three subunits, lung phosphofructokinase consists of many different isozymes and we might expect a very complex regulation of phosphofructokinase activity; (ii) the increase in phosphofructokinase specific activity in the period before birth [7] is not associated with a change in subunit composition; (iii) a difference in regu- lation of phosphofructokinase activity on the basis of subunit composition during lung development is not to be expected.

Although we did observe a difference in phosphofructokinase activation by glucose 1,6-bisphosphate at different stages of development (Fig. 6) it is questionable whether this relative small difference has physiological meaning. These stimulation experiments were performed to show the susceptibility of lung phosphofructokinase to activation by glucose 1,6-bisphosphate and fructose 2,6-bisphosphate under conditions in vitro where phosphofructokinase is sensitive to regulation. On the other hand, there seems to be no difference in phosphofructokinase activation by fructose 2,6-bisphosphate (Fig. 5) during lung development and the results suggest that lung phosphofructokinase has a much higher affinity for fructose 2,6-bisphosphate compared to glucose 1,6-bisphosphate. These findings correspond with those found for muscle [17] and liver [19] phosphofructokinase.

The absolute amount of glucose 1,6-bisphosphate in the rat lungs increases dramatically after birth with a maximum of approx. $1.0 \mu \mathrm{mol} / \mathrm{g}$ protein at 2 days after birth. As both glycogen breakdown and the acceleration of surfactant synthesis take place in the last 2 days of gestation there seems to be no correlation between the time of maximal glucose 1,6-bisphosphate content and the time of glycogen breakdown and onset of surfactant synthesis. It should be noted, however, that the rate of surfactant phosphatidylcholine synthesis and the accumulation of this lipid in the alveolar spaces reach maximal values almost coinciding with the glucose 1,6-bisphosphate level [33]. We found in adult rat lung $21 \mu \mathrm{mol}$ glucose 1,6-bisphosphate/ $\mathrm{kg}$ wet weight.

The time of maximal fructose 2,6-bisphosphate content in rat lung does correspond with the time of glycogen breakdown and the acceleration of surfactant synthesis in the rat lung. The concentration of glucose 1,6-bisphosphate found in rat lung during perinatal development varies from $13.5 \mu \mathrm{M}$ to $55.0 \mu \mathrm{M}$ and that of fructose 2,6-bisphosphate from $0.29 \mu \mathrm{M}$ to $2.0 \mu \mathrm{M}$. These concentrations were calculated assuming that on average $83 \%$ of the wet weight consists of water [34]. The half maximal activation of lung phosphofruc- 
tokinase in our assay conditions takes place at roughly $0.25 \mu \mathrm{M}$ fructose 2,6-bisphosphate and 50 $\mu \mathrm{M}$ glucose 1,6-bisphosphate. That would suggest that in those rat lungs which have high levels of glucose 1,6-bisphosphate, phosphofructokinase is activated and that in all rat lungs during perinatal development phosphofructokinase is activated by fructose 2,6-bisphosphate. However, the experimental conditions used do not reflect the in vivo situation and especially the effect of hexose bisphosphates on phosphofructokinase is very dependent on the experimental conditions [19].

Another physiological consequence of the changes in fructose 2,6-bisphosphate (and possibly glucose 1,6-bisphosphate) levels in rat lung might be found in a very recent discovery of a phosphofructokinase inactivating protein by Brand and Söling [35]. The inactivation of phosphofructokinase by this inactivating protein results in dissociation of the enzyme to inactive protomers and has been demonstrated for liver, muscle and brain. Fructose 2,6-bisphosphate and fructose 1,6-bisphosphate inhibit the inactivating protein; the authors suggest that changes in concentration of fructose 2,6-bisphosphate or fructose 1,6-bisphosphate (and possibly glucose 1,6-bisphosphate) might regulate phosphofructokinase activity in this way in addition to their importance as positive allosteric effectors of phosphofructokinase activity.

In conclusion, the acceleration of surfactant synthesis might well be regulated at the glycolytic level by fructose 2,6-bisphosphate activation of phosphofructokinase. The onset of this process seems neither to be regulated by a change in glucose 1,6-bisphosphate nor by a change in subunit composition of phosphofructokinase during perinatal development.

However, it has to be considered that synthesis of lung surfactant takes place in type II alveolar pneumocytes. Fetal lungs consist, depending on their gestational age, of $20-45 \%$ type II pneumocytes [36]. Preliminary results demonstrated that all three subunits of phosphofructokinase are expressed in the type II cell and we are now investigating the activities of glycolytic enzymes and their effectors in the isolated type II alveolar pneumocytes.

\section{Acknowledgements}

The investigation described in this paper was supported in part by the Netherlands Foundation for Chemical Research (S.O.N). We greatly appreciate the generous help of Dr. G.A. Dunaway and T.P. Kasten in setting up this study. The authors thank Batja van de Pol for typing the manuscript.

\section{References}

1 Goerke, J. (1974) Biochim. Biophys. Acta 344, 241-261

2 Possmayer, F. (1984) in Pulmonary Surfactant (Robertson, B., Van Golde, L.M.G. and Batenburg, J.J., eds.), pp. 295-355, Elsevier, Amsterdam

3 Kikkawa, Y., Motoyama, E.K. and Gluck, L. (1968) Am. J. Pathol. 52, 177-192

4 Buckingham, S., Heinemann, H.O., Sommers, S.C. and McNarry, W.F. (1966) Am. J. Pathol. 48, 1027-1041

5 Maniscalco, W.M., Wilson, C.M., Gross, I., Gobran, L., Rooney, S.A. and Warshaw, J.B. (1978) Biochim. Biophys. Acta 530, 333-346

6 Bourbon, J.R., Rieutort, M., Engle, M.J. and Farrell, P.M. (1982) Biochim. Biophys. Acta 712, 382-389

7 Rijksen, G., Staal, G.E.J., Streefkerk, M., De Vries, A.C.J., Batenburg, J.J., Heesbeen, E.C. and Van Golde, L.M.G. (1985) Biochim. Biophys. Acta 838, 114-121

8 Foe, L.G. and Kemp, R.G. (1984) Arch. Biochem. Biophys. $228,503-511$

9 Dunaway, G.A. and Kasten, T.P. (1985) J. Biol. Chem. 260, $4180-4185$

10 Kahn, A., Meienhofer, M.-C., Cottreau, D., Lagrange, J.-L. and Dreyfus, J.-C. (1979) Hum. Genet. 48, 93-108

11 Vora, S. (1981) Blood 57, 724-732

12 Krzanowski, J. and Matschinsky, F.M. (1969) Biochem. Biophys. Res. Commun. 34, 816-823

13 Rapoport, S. (1974) in The Human Red Cell In Vitro (Greenwalt, T.J. and Jamieson, G.A., eds.), pp. 153-178 Grune \& Stratton, New York

14 Rose, I.A. and Warms, J.V.B. (1974) Biochem. Biophys. Res. Commun. 59, 1333-1340

15 Rijksen, G. and Staal, G.E.J. (1977) FEBS Lett. 80, 61-65

16 Uyeda, K., Furuya, E. and Luby, L.J. (1981) J. Biol. Chem. 256, 8394-8399

17 Foe, L.G., Latshaw, S.P. and Kemp, R.G. (1983) Biochemistry $22,4601-4606$

18 Van Schaftingen, E., Hue, L. and Hers, H.-G. (1980) Biochem. J. 192, 897-901

19 Van Schaftingen, E., Jett, M.-F., Hue, L. and Hers, H.-G. (1981) Proc. Natl. Acad. Sci. USA 78, 3483-3486

20 Hue, L. and Bartrons, R. (1984) Biochem. J. 218, 165-170

21 Loiseau, A.M., Rousseau, G.G. and Hue, L. (1985) Cancer Res. 45, 4263-4269

22 Bruni, P., Farnararo, M., Vasta, V. and D'Alessandro, A. (1983) FEBS Lett. 157, 39-42

23 Malaisse, W.J., Malaisse-Lagae, F. and Sener, A. (1982) Diabetologia 23, 1-5 
24 Laemmli, U.K. (1970) Nature 277, 680-685

25 Wray, W., Boulikas, T., Wray, V.P. and Hancock, R. (1981) Anal. Biochem. 118, 197-203

26 Hawkes, R. (1982) Anal. Biochem. 123, 143-146

27 Gerber, G., Winczuk, E. and Rapoport, S. (1973) Acta. Biol. Med. Germ. 6, 759-771

28 Bradford, M.M. (1976) Anal. Biochem. 72, 248-254

29 Dunaway, G.A. and Kasten, T.P. (1985) J. Mol. Cell. Cardiol. 17, 947-957

30 Meienhofer, M.-C., Cottreau, D., Dreyfus, J.-C. and Kahn, A. (1980) FEBS Lett. 110, 219-222
31 Foe, L.G. and Kemp, R.G. (1985) J. Biol. Chem. 260, 726-730

32 Tsai, M.Y. and Kemp, R.G. (1973) J. Biol. Chem. 248, $785-792$

33 Rooney, S.A. (1984) Env. Health Perspect. 55, 205-266

34 Sauer, L.A. and Dauchy, R.T. (1986) Cancer Res. 46, 689-693

35 Brand, I.A. and Söling, H.-D. (1986) J. Biol. Chem. 261, $5892-5900$

36 Adamson, I.Y.R. and King, G.M. (1984) Am. Rev. Respir. Dis. $129,130-134$ 